\title{
Protein overexpression of toll-like receptor 4 and myeloid differentiation factor 88 in oral squamous cell carcinoma and clinical significance
}

\author{
LILI LI ${ }^{1-5}$, ZHUOQIAN ZHOU ${ }^{1-4}$, KHANGVU MAI $^{1-4}$, PING LI $^{6}$, ZONGQI WANG $^{1-4}$, YAXI WANG $^{1-4}$, \\ YANG CAO $^{6}$, XUEMENG MA ${ }^{6}$, TINGTING $\mathrm{ZHANG}^{7}$ and DAIYOU WANG ${ }^{1-4}$
}

\begin{abstract}
${ }^{1}$ Department of Oral and Maxillofacial Surgery; ${ }^{2}$ Guangxi Key Laboratory of Oral and Maxillofacial Rehabilitation and Reconstruction; ${ }^{3}$ Guangxi Key Laboratory of Oral and Maxillofacial Surgery Disease Treatment; ${ }^{4}$ Guangxi Clinical Research Center for Craniofacial Deformity, College and Hospital of Stomatology, Guangxi Medical University, Nanning, Guangxi 530021; ${ }^{5}$ Department of Stomatology, Maternal and Child Health Hospital of Guangxi Zhuang Autonomous Region, Nanning, Guangxi 530028; Departments of ${ }^{6}$ Pathology and ${ }^{7}$ Disease Control and Prevention, College and Hospital of Stomatology, Guangxi Medical University, Nanning, Guangxi 530021, P.R. China
\end{abstract}

Received January 29, 2021; Accepted July 13, 2021

DOI: 10.3892/ol.2021.13047

\begin{abstract}
Oral squamous cell carcinoma (OSCC) is the most common type of malignancy of the head and neck. In the present study, the expression of Toll-like receptor 4 (TLR4) and myeloid differentiation primary response gene 88 (MyD88) was evaluated in 55 OSCC tissues and their corresponding adjacent tissues using immunohistochemistry and reverse-transcription quantitative PCR. The results indicated that TLR4 and MyD88 were overexpressed in OSCC. Furthermore, high expression of MyD88 was negatively associated with a poor degree of differentiation, recurrence and metastasis of the tumor and was positively associated with underlying disease, including hypertension, heart disease and diabetes mellitus. Furthermore, high expression of TLR4 was positively associated with a long growth time of the tumor. In conclusion, the present study evaluated the expression levels of TLR4 and MyD88 in OSCC, as well as the association between them and clinicopathological factors, to provide markers for the prognosis and treatment of OSCC. These two genes may serve as biomarkers to optimize OSCC treatment, setting a new direction for stratifying patients and developing precise and personalized treatment regimens; the TLR4/MyD88 pathway may serve as a potential therapeutic target in the future.
\end{abstract}

Correspondence to: Dr Daiyou Wang, Department of Oral and Maxillofacial Surgery, College and Hospital of Stomatology, Guangxi Medical University, 10 Shuangyong Road, Nanning, Guangxi 530021, P.R. China

E-mail: wangdaiyou@sina.com

Key words: MyD88, TLR4, oral squamous cell carcinoma, immunohistochemistry, reverse-transcription quantitative PCR, prognosis

\section{Introduction}

Oral cancer is a malignant tumor type that occurs in the lips or mouth (1). $90 \%$ of oral cancers are histologically originated from squamous cells (2) and oral cancer has traditionally been defined as oral squamous cell carcinoma (OSCC) (3). According to US government statistics, OSCC is the eighth most common cancer type among males and the 14th most common cancer type among females (4). The development of OSCC is a complex process modulated by genetic and environmental factors. The accumulation of multiple genetic alterations is regulated by hereditary predisposition, including sex and ethnicity. Despite recent advances in imaging, surgery, radiation and systemic therapy, the overall survival of patients with OSCC has not significantly improved over the past 20 years (5). Therefore, OSCC remains a major clinical challenge and there are currently no available biomarkers to guide treatment decisions. Identifying reliable biomarkers and novel molecular targets is critical to the stratification of patients to develop precise and personalized treatment regimens, helping physicians to deliver early and appropriate treatment regimens in a timely manner that may reduce the risk of squamous cell cancer recurrence (6). Numerous biomolecules are involved in OSCC development and may provide references for treatment and prognosis (6).

Toll-like receptor 4 (TLR4), a transmembrane protein located on chromosome 9q32-q33, is a member of the TLR protein family that has been well studied. TLR4 has a fundamental role in various functions, including defense against pathogens, activation of innate immunity and regulation of chronic inflammation. Previous studies have indicated that stimulation of TLR4 expression on tumor cells may induce chronic inflammation and promote tumor growth (7). Overexpression of TLR4 has been observed in several types of human cancer, such as breast (8), melanoma (9), colon (10), ovarian (11) and prostate cancers (10).

MyD88 is a central regulator of innate immunity; it acts directly downstream of TLRs and cytokine receptors, while 
also associated with carcinogenesis (12). MyD88 activates the TLRs or interleukin-1 receptor (IL-1Rs) pathways autonomously, or perhaps in relation to TNF receptor-associated factor 6 signaling, allowing tumor cells to proliferate indefinitely (3). MyD88 is involved in oncogene-induced inflammation and contributes to the development of cancers of the skin, liver, pancreas and colon, as well as sarcoma formation (13), and its expression is associated with poor prognosis in colorectal cancer (14). Other studies have indicated that MyD88, coupled with TLR4, has an essential role in skin tumor promotion (3). Previous studies have indicated that activation of the TLR4/MyD88-mediated signaling pathway promotes tumor occurrence and metastasis $(15,16)$. MyD88 also has a mucosal protective effect, involving downstream IL-18R for mucosal repair during oncogenic virus carcinogenesis or during azomethane/sodium dextran sulfate-induced colon cancer (8).

OSCC is a preventable disease and its risk factors and clinical relevance have been well documented (4); effective prevention and treatment may improve the prognosis of OSCC. The present study aimed to integrate the clinical and histological features as well as the expression of TLR4 and MyD88 in OSCC to verify the overexpression of TLR4 and MyD88. Furthermore, it aimed to explore the association between the development of oral cancer and the expression levels of TLR4 and MyD88 to elucidate the biological mechanisms of OSCC.

\section{Materials and methods}

Patients. The electronic medical record system of the School of Stomatology, Guangxi Medical University (Nanning, China), was searched to retrieve information on the cases of OSCC encountered at the Department of Oral and Maxillofacial Surgery from January 2020 to September 2020.

The inclusion criteria were as follows: i) The patients were diagnosed with OSCC by pathological examination; ii) patients underwent radical resection of oral cancer; iii) all patients were initially treated and had no preoperative history of biological immunotherapy or other malignant tumors. In addition, the following exclusion criteria were applied: i) Patients with another primary cancer or multiple primary cancers in the past or present; ii) cases accompanied with immune system diseases; iii) patients who objected to the use of their tumor specimens for the present study; iv) patients accompanied by acute or chronic infection, with long-term allergy. The degree of differentiation of a patient's malignant tumor was derived from the patient's discharge diagnosis, which was issued by two pathologists from the Department of Pathology of the Stomatological Hospital Affiliated with Guangxi Medical University (Nanning, China). Clinical and follow-up data were recorded for all included patients. There were 39 males and 16 females, with a median age of 56 years (mean \pm standard deviation; $54.64 \pm 12.87$ years; range, 27-79 years). The mean follow-up time was 4 months (range, 1-9 months). The present study was approved by the Ethics Committee of the School of Stomatology, Guangxi Medical University (Nanning, China) and all patients enrolled provided written informed consent. Tumor growth time can be inferred from the patient's clinical admission record.
Specimen collection. For reverse-transcription quantitative $\mathrm{PCR}$, the removed tumor tissues and corresponding adjacent paracancerous tissues from patients with OSCC who underwent surgery on the same day were collected at the surgical operating room of the Stomatological Hospital Affiliated to Guangxi Medical University (Nanning, China) and were stored in RNA Keeper for later reverse transcription-quantitative (RT-q)PCR analysis.

For immunohistochemistry, the histological specimens of the 55 corresponding cases of OSCC were then formalin-fixed and paraffin-embedded and stored at the Department of Pathology for later analysis by immunohistochemistry. A retrospective immunohistochemical analysis was performed on 55 formalin-fixed paraffin-embedded specimens of clinically and histologically confirmed cases of OSCC and corresponding adjacent non-tumoral tissues that had been examined and stored at the Department of Pathology, the Department of Oral and Maxillofacial Surgery, College of Stomatology, Guangxi Medical University (Nanning, China).

Immunohistochemistry. The tissue sections were first dewaxed in gradient xylene and then rehydrated with an alcohol gradient. After rinsing with tap water and soaking in pure water, the sections were immersed in sodium citrate buffer (Beijing Zhongshan Golden Bridge Biotechnology, Beijing, China) and microwave-heated for $5 \mathrm{~min}$ to retrieve antigen. After the slices were cooled, they were rinsed with pure water 3 times and then $0.3 \%$ hydrogen peroxide was used to block endogenous peroxidase activity. Next, anti-TLR4 polyclonal antibody and anti-MyD88 antibody (1:100 dilution; Bioss; cat. nos. bs-1021R-50 and bs-1047R-50) were applied to the tissue sections with incubation overnight in the refrigerator at $4^{\circ} \mathrm{C}$. Biotinylated goat anti-rabbit $\mathrm{IgG}$ (does not need to be diluted; ZSGB-BIO; PV-6000) was then dripped on tissue sections and they were incubated at $37^{\circ} \mathrm{C}$ for $20 \mathrm{~min}$. The sections were washed with PBS three times between all steps for 3 min each time. The sections were then stained with diaminobenzidine for $2 \mathrm{~min}$ to visualize the antibodies and counterstained with hematoxylin for $1 \mathrm{~min}$. The tissue sections were then dehydrated with ethanol and mounted with glass slips.

The expression of TLR4 and MyD88 was examined in 5 different visual fields under a 20X enlargement factor microscope. Each section stained was observed and evaluated by two independent investigators. Sections were scored by cytoplasmic staining intensity and distribution. The staining intensity was rated as follows: 0 , no staining; 1 , weak staining; 2 , moderate staining; 3 , strong staining. The staining distribution was divided into 4 grades according to the percentage of tumor cells stained: $0,0 \% ; 1,<25 \% ; 2,25-50 \% ; 3,51-75 \%$; 4 , $>75 \%$ (17). The final staining scores were calculated as follows: Staining intensity multiplied by the score for the percentage of stained tumor cells. A final staining score of $>3$ was considered to indicate positive protein overexpression.

$R T$-qPCR analysis. Total RNA was isolated from human OSCC tissues and paracancerous tissues using TRIzol ${ }^{\circledR}$ reagent (Takara Bio, Inc.) according to the manufacturer's protocol. Total RNA (1 mg) was reverse transcribed to cDNA with a PrimeScript RT reagent Kit (Takara Bio, Inc.). The 
temperature and duration of reverse transcription were $42^{\circ} \mathrm{C}$ for $2 \mathrm{~min}$, followed by $37^{\circ} \mathrm{C}$ for $15 \mathrm{~min}$ and $85^{\circ} \mathrm{C}$ for $5 \mathrm{sec}$. qPCR was performed with the $2 *$ RealStar Green Fast Mixture (Genebrick) using the following thermocycling program: Pre-denaturation at $95^{\circ} \mathrm{C}$ for $2 \mathrm{~min}$, followed by 40 cycles of $95^{\circ} \mathrm{C}$ for $15 \mathrm{sec}, 60^{\circ} \mathrm{C}$ for $15 \mathrm{sec}$ and $72^{\circ} \mathrm{C}$ for $45 \mathrm{sec}$, and a final extension at $72^{\circ} \mathrm{C}$ for $30 \mathrm{sec}$. The primer sequences were as follows: TLR4 forward, 5'-CCAAGAACCTGGACC TGAGCTTTA-3' and reverse 5'-CCATCTTCAATTGTCTGG ATTTCAC-3'; MyD88 forward, 5'-AGCCAGGCTGGAGCA AGGTA-3' and reverse, 5'-GGCAGCTAAATGCCTCAA CAAGA-3'; GAPDH forward, 5'-GCACCGTCAAGGCTG AGAAC-3' and reverse, 5'-TGGTGAAGACGCCAGTGGA-3'. Quantifications were performed using the $\Delta \Delta \mathrm{Cq}$ method (18), where GAPDH was used as a reference gene for normalization of RNA expression.

Statistical analysis. SPSS 20 software (IBM Corporation) was used for statistical analysis. To examine the association of the immunohistochemical results with the pathological features and clinical outcomes of patients, Pearson's $\chi^{2}$ test was used (Table SI). For the RT-qPCR results, the t-test was used to investigate the possible association between mRNA expression and the pathological and clinical results of the cases being studied (Table SII). For comparisons of $>2$ items, single factor analysis of variance was used, bonferroni correction was performed to mitigate variability when comparing multiple samples. Spearman's bivariate correlation method was used to detect the correlation between TLR4 and MyD88 expression in OSCC tissues (Table I). $\mathrm{P}<0.05$ was considered to indicate statistical significance.

\section{Results}

Clinical and pathological features. All 55 patients were clinically and histologically confirmed to have primary OSCC. The patients' age ranged from 27 to 79 years, with an average age of 54 years at first diagnosis. Among the 55 patients, 39 were males and 16 were females. Sections were examined independently by two pathologists. Well-differentiated OSCC is similar to normal squamous epithelium, with varying numbers of basal cells and squamous cells with intercellular bridge, obvious keratosis, a small amount of mitotic figures, low frequency of atypical mitotic figures and multinucleated cells and no obvious pleomorphism of nuclei and cells. Moderately-differentiated OSCC has a unique nuclear pleomorphism and mitotic features, including abnormal mitotic features, infrequent keratosis and inconspicuous intercellular bridging. Poorly-differentiated OSCC is dominated by immature cells with a large number of normal or abnormal mitotic images, a low frequency of keratinization and almost no intercellular bridging (18). Histologically, the 55 patients were classified as well and moderately differentiated (51 cases) and poorly differentiated (4 cases). TNM staging was performed according to the Union for International Cancer Control, according to which 6 cases were stage I, 16 cases were stage II, 20 cases were stage III and 13 cases were stage IV (19). No lymph node metastasis was detected in 29 patients and lymph node metastasis was confirmed in 26 patients. The last follow-up ranged from 1 to 9 months (mean, 4 months).
Recurrence was reported in 2 cases. A total of 12 cases were basic disease-negative and 43 cases were basic disease-positive. Underlying diseases mainly included diabetes, hypertension and heart disease.

Histological localization of TLR4 and MyD88. Immunohistochemical staining of intact paraffin sections of OSCC tissues and paracancerous tissues was performed using standard procedures. In oral squamous cells, it was observed that TLR4 was localized in the membrane and cytoplasm, while MyD88 was localized only in the cytoplasm. TLR4 and MyD88 were indicated to be expressed in paracancerous tissues but the expression was weak. In addition, it was revealed that TLR4 and MyD88 were highly expressed in inflammatory cells and duct epithelium.

TLR4 and MyD88 are highly expressed in OSCC. Two pathologists separately evaluated all specimens using the same staining scoring criteria. TLR4 and MyD88 protein expression was analyzed in all 55 samples. The expression results of TLR4 and MyD88 were as follows: TLR4 and MyD88 were rarely expressed in paracancerous tissues, while positive staining was observed in most of the 55 OSCC specimens. In the OSCC area, staining for TLR4 was positive in 49 cases and negative in 6 cases. Staining for TLR4 in their corresponding adjacent tissues was positive in 2 cases and negative in 53 cases and the difference from the tumor tissues was statistically significant $\left(\chi^{2}=80.754, \mathrm{P}<0.001\right)$. In OSCC, staining for MyD88 was positive in 46 cases and negative in 9 cases. In normal adjacent tissues, staining for MyD88 was positive in 6 cases and negative in 49 cases and the difference from the tumor tissues was statistically significant $\left(\chi^{2}=58.355, \mathrm{P}<0.001\right.$; Table II). The RT-qPCR analysis also revealed elevated expression of TLR4 and MyD88 in OSCC (Table I). The results of the RT-qPCR analysis were consistent with those of the immunohistochemical detection in terms of TLR4 and MyD88 being highly expressed in OSCC, while their levels in the corresponding tissue adjacent to carcinoma of TLR4 and MyD88 were low (Table I, Figs. 1-3). Therefore, it was concluded that the expression levels of TLR4 and MyD88 in OSCC were higher than the corresponding paracancerous tissues.

MyD88 is highly expressed in OSCC with a high and intermediate degree of differentiation compared with that in poorly differentiated OSCC. The positive expression rate of MyD88 in OSCC was compared among different histological grades. It was revealed that the expression rate of MyD88 was higher in well and moderately differentiated tumors than in poorly differentiated tumors ( $\mathrm{P}=0.01$; Table III, Fig. 2). This result suggested that overexpression of MyD88 may be related to a higher degree of differentiation.

MyD88 is highly expressed in patients without short-term recurrence. The expression of MyD88 was compared between OSCC tissues of patients with or without short-term recurrence (within a year). The expression of MyD88 was high in patients without short-term recurrence, while it was low in patients with recurrence $(\mathrm{P}=0.022$; Table III). However, there were only 2 cases of recurrence, which was not sufficient to 
Table I. mRNA expression of MyD88 and TLR4 in OSCC and pericarcinomatous tissue.

\begin{tabular}{|c|c|c|c|c|c|c|c|}
\hline Tissue type & $\mathrm{N}$ & Myd88 & $\mathrm{t} / \mathrm{F}$ & P-value & TLR4 & $\mathrm{t} / \mathrm{F}$ & P-value \\
\hline OSCC & 55 & $3.941 \pm 9.344$ & 2.327 & 0.024 & $2.401 \pm 4.068$ & 2.545 & 0.014 \\
\hline Pericarcinomatous tissue & 55 & $1.012 \pm 0.036$ & & & $1.005 \pm 0.006$ & & \\
\hline
\end{tabular}

Values are expressed as the mean \pm standard deviation. The results were obtained by reverse transcription-quantitative PCR; quantification was performed using the $\triangle \triangle \mathrm{Cq}$ method with GAPDH used as a reference gene for normalization of mRNA expression. $\mathrm{P}<0.05$ determined with the paired t-test was considered to indicate a statistically significant difference. TLR4, Toll-like receptor 4; MyD88, myeloid differentiation primary response gene 88 ; OSCC, oral squamous cell carcinoma.

Table II. Protein expression of MyD88 and TLR4 in OSCC and pericarcinomatous tissue.

\begin{tabular}{|c|c|c|c|c|c|c|c|c|}
\hline \multirow[b]{2}{*}{ Tissue type } & \multicolumn{2}{|c|}{ Myd88 } & \multirow[b]{2}{*}{$\chi^{2}$} & \multirow[b]{2}{*}{ P-value } & \multicolumn{2}{|c|}{ TLR4 } & \multirow[b]{2}{*}{$\chi^{2}$} & \multirow[b]{2}{*}{ P-value } \\
\hline & - & + & & & - & + & & \\
\hline OSCC & $9(16.3)$ & $46(83.7)$ & 58.355 & $<0.001$ & $6(10.9)$ & $49(89.1)$ & 80.754 & $<0.001$ \\
\hline Pericarcinomatous tissue & $49(89.1)$ & $6(10.9)$ & & & $53(96.4)$ & $2(3.6)$ & & \\
\hline
\end{tabular}

Values are expressed as $\mathrm{n}(\%)$. Results were obtained from immunohistochemical examination and analyzed by Pearson's $\chi^{2}$ test. P $<0.05$ was considered to indicate statistical significance. TLR4, Toll-like receptor 4; MyD88, myeloid differentiation primary response gene 88; OSCC, oral squamous cell carcinoma.
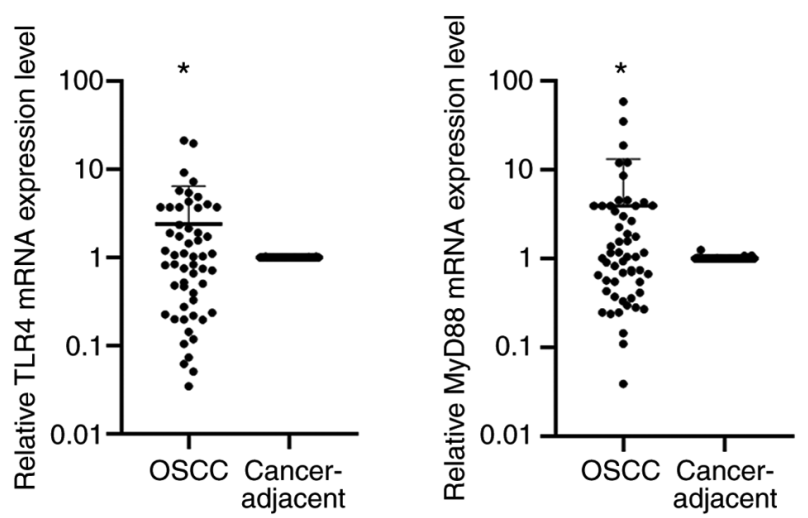

Figure 1. RT-qPCR analysis revealed higher expression of TLR4 and MyD88 in OSCC than in corresponding paracancerous tissues. The mRNA expression level of TLR4 and MyD88 was detected by SYBR Green I reverse transcription-quantitative PCR and quantifications were performed using the $\Delta \Delta \mathrm{Cq}$ method, where GAPDH was used as a reference gene for normalization of RNA expression. "P<0.05 vs. Cancer-adjacent tissue. RT-qPCR, reverse transcription-quantitative PCR; TLR4, Toll-like receptor 4; MyD88, myeloid differentiation primary response gene 88 ; OSCC, oral squamous cell carcinoma.

signify that the overexpression of MyD88 is related to the prognosis of OSCC.

MyD88 is highly expressed in patients with underlying disease. The expression of MyD88 in OSCC tissues of patients with or without underlying diseases was then compared. It was revealed that the expression of MyD88 was high in patients with underlying disease, while it was low in patients without underlying disease $(\mathrm{P}=0.025$; Table III). This result indicated that overexpression of MyD88 may be related to underlying disease. The protein and mRNA expression levels of MyD88 in OSCC tissues were not associated with to sex, age, ethnicity, smoking, alcohol consumption, tumor stage,nodal status,tumor site,TNM stage,contralateral lymph node metastasis and diabetes(Tables SI and SII).

TLR4 expression is significantly associated with tumor growth time. The RT-qPCR results in Table IV indicated that the expression level of TLR4 was significantly associated with the tumor growth time $(\mathrm{P}=0.001)$. A longer growth time of OSCC was associated with a higher expression of TLR4 protein; however, MyD88 was not associated with tumor growth time. The protein and the mRNA expression of TLR4 in OSCC tissues of patients are unconcerned with gender, age, nationality, smoking or drinking and so on (Tables SI and SII).

TLR4 is positively correlated with MyD88 expression in OSCC. Spearman's correlation analysis was performed to calculate the correlation between the protein and mRNA expression levels of TLR4 and MyD88. Analysis of the immunohistochemical results indicated a positive correlation between TLR4 and MyD88 protein expression levels in OSCC $(\mathrm{r}=0.653, \mathrm{P}<0.001)$. Furthermore, the RT-qPCR results also revealed a positive correlation between TLR4 and MyD88 mRNA expression levels ( $\mathrm{r}=0.431, \mathrm{P}=0.001)$. In addition, the expression of MyD88 in OSCC was higher than that in TLR4 $(\mathrm{t}=7.361, \mathrm{P}<0.001)$. These statistical results indicated that because MyD88 is downstream of TLR4 (20), upregulation of TLR4 expression promoted the upregulation of MyD88 expression. 

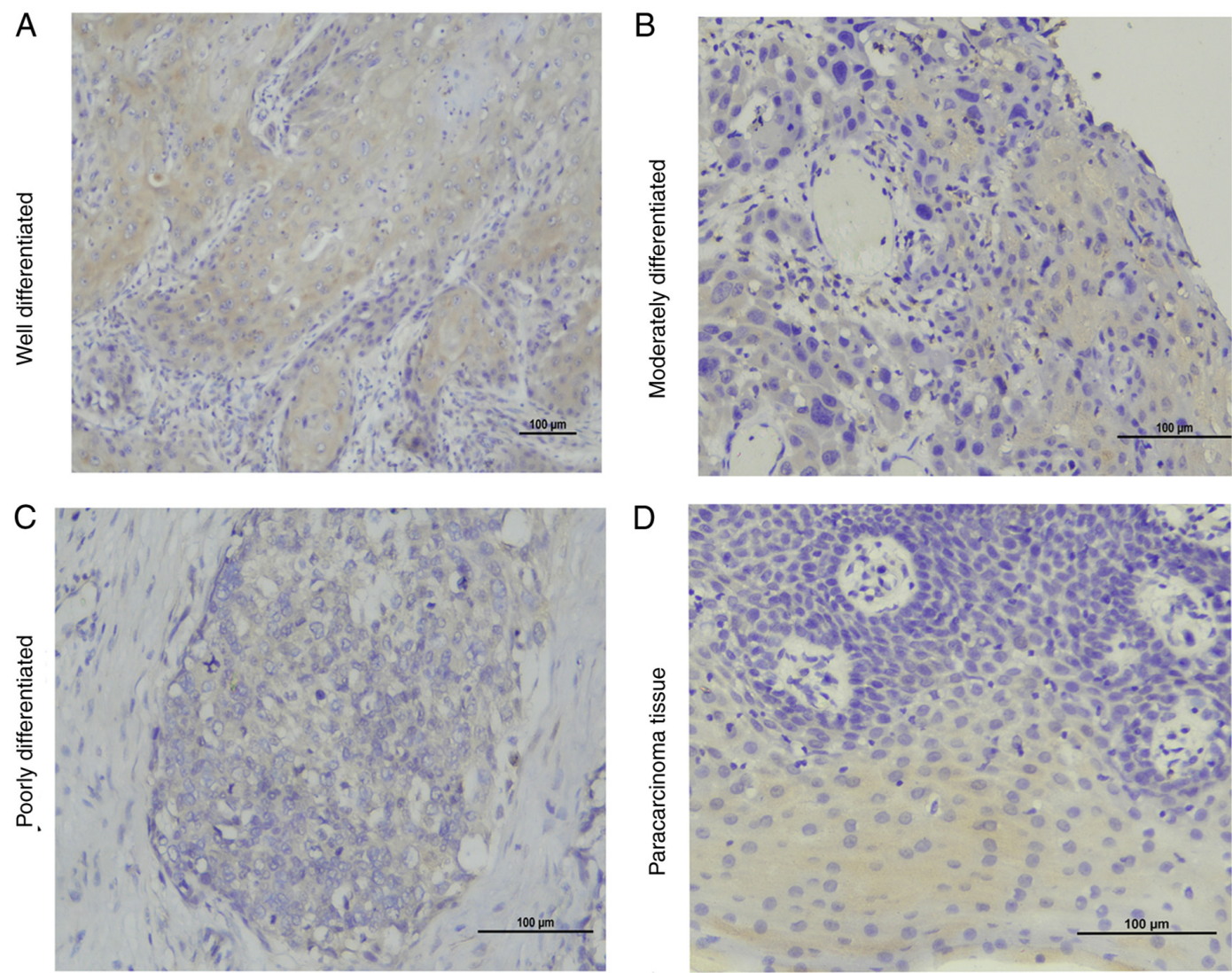

Figure 2. Immunohistochemical staining for MyD88 indicated positive expression in oral squamous cell carcinoma; positive cells are yellow or brown (anti-MyD88 antibody diluted 1:100, original magnification, x20; scale bar, $100 \mu \mathrm{m}$ ). Degree of differentiation: (A) Well, (B) moderate, (C) poor; (D) paracarcinoma tissue. MyD88, myeloid differentiation primary response gene 88.
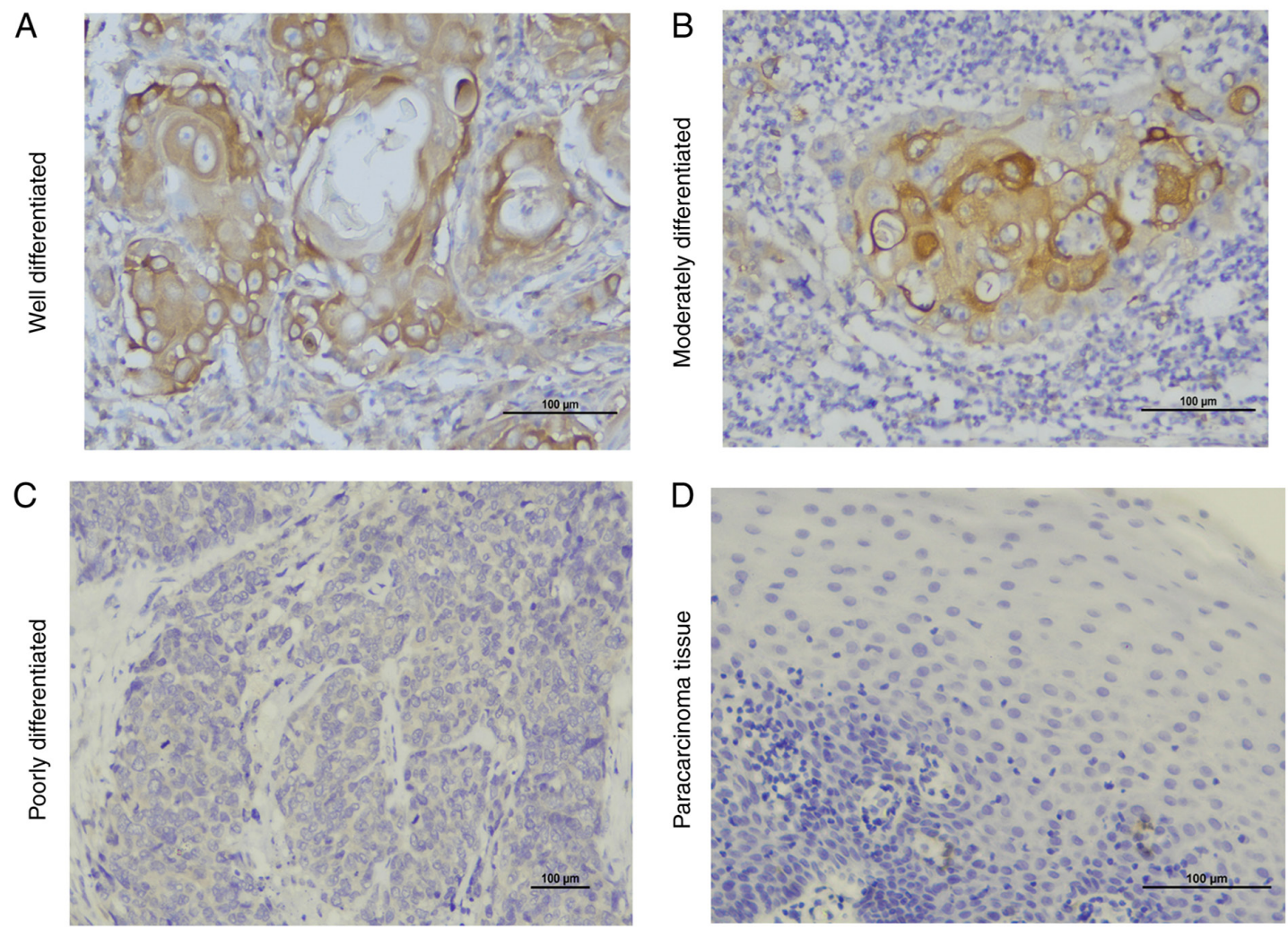

Figure 3. Immunohistochemical staining of TLR4 indicated positive expression in oral squamous cell carcinoma; positive cells are yellow or brown (anti-TLR4 antibody diluted 1:100; original magnification, x20; scale bar, $100 \mu \mathrm{m}$ ). Degree of differentiation: (A) Well, (B) moderate, (C) poor; (D) paracarcinoma tissue. TLR4, Toll-like receptor 4. 
Table III. Association between the clinicopathological features and the protein expression of TLR4 and MyD88.

\begin{tabular}{|c|c|c|c|c|c|c|c|c|c|}
\hline \multirow[b]{2}{*}{ Characteristic } & \multirow[b]{2}{*}{$\mathrm{N}$} & \multicolumn{2}{|c|}{ Myd88 } & \multirow[b]{2}{*}{$\chi^{2}$} & \multirow[b]{2}{*}{ P-value } & \multicolumn{2}{|c|}{ TLR4 } & \multirow[b]{2}{*}{$\chi^{2}$} & \multirow[b]{2}{*}{ P-value } \\
\hline & & - & + & & & - & + & & \\
\hline Pathological differentiation grade & & & & 6.709 & 0.01 & & & 0.000 & 1.000 \\
\hline Well-moderate & 51 & $6(11.8)$ & $45(88.2)$ & & & $6(11.8)$ & $45(88.2)$ & & \\
\hline Poor & 4 & $3(75.0)$ & $1(25.0)$ & & & $0(0.0)$ & $4(100)$ & & \\
\hline Underlying disease & & & & 5.010 & 0.025 & & & 0.718 & 0.153 \\
\hline No & 12 & $5(41.7)$ & $7(58.3)$ & & & $0(0.0)$ & $12(100.0)$ & & \\
\hline Yes & 43 & $4(9.3)$ & $39(90.7)$ & & & $6(14.0)$ & $37(86.0)$ & & \\
\hline Recurrence & & & & 5.214 & 0.022 & & & 0.000 & 1.000 \\
\hline No & 53 & $7(13.2)$ & $46(86.8)$ & & & $6(11.3)$ & $47(88.7)$ & & \\
\hline Yes & 2 & $2(100.0)$ & $0(0.0)$ & & & $0(0.0)$ & $2(100.0)$ & & \\
\hline
\end{tabular}

Values are expressed as n (\%). Underlying diseases mainly include hypertension, diabetes and heart disease. Recurrent cases were collected until October 2020. P-values are from a continuous correction algorithm. TLR4, Toll-like receptor 4; MyD88, myeloid differentiation primary response gene 88 .

Table IV. Association between the clinicopathological features and the mRNA expression of TLR4 and MyD88.

\begin{tabular}{lrcccccr}
\hline Tumor growth time (months) & $\mathrm{N}$ & $\mathrm{Myd} 88$ & $\mathrm{t} / \mathrm{F}$ & $\mathrm{P}$-value & TLR4 & $\mathrm{t} / \mathrm{F}$ & P-value \\
\hline$\leq 6$ (A) & 42 & $3.930 \pm 10.310$ & 0.011 & 0.989 & $1.430 \pm 1.850$ & 7.688 & 0.001 \\
$6-12$ (B) & 4 & $3.380 \pm 1.800$ & & & $2.920 \pm 2.270$ & & \\
$12-18(\mathrm{C})$ & 9 & $4.220 \pm 6.590$ & & & $6.650 \pm 8.150$ & & \\
\hline
\end{tabular}

Values are expressed as the mean \pm standard deviation. P-values are from single-factor ANOVA with Bonferroni correction. Between-group comparisons for MyD88: A vs. B, P=1.000; A vs. C, $\mathrm{P}=1.000$; B vs. C, $\mathrm{P}=1.000$. For TLR4: A vs. $\mathrm{B}, \mathrm{P}=1.000$; A vs. C, $\mathrm{P}=0.001$; B vs. C, $\mathrm{P}=0.281$. TLR4, Toll-like receptor 4; MyD88, myeloid differentiation primary response gene 88 .

\section{Discussion}

OSCC is a global public health issue and a particular challenge for oral physicians. The major complaints associated with OSCC are impairment of speech, swallowing and chewing functions, with pain as the major symptom. According to the International Agency for Research on Cancer, OSCC has a high incidence rate globally, with $>300,000$ cases diagnosed each year and $\sim 145,000$ deaths annually (21). Approximately one-third of patients with OSCC are diagnosed with stage I/II disease (22). These patients have a good prognosis, with a cure rate of $\sim 80 \%$ (stage I) and 65\% (stage II) (23). However, two-thirds of OSCC cases are diagnosed at the late stages of the disease (stage III or IV) (24), with a 5 -year survival rate of $<50 \%$ and a cure rate of $30 \%$ (25). Therefore, early diagnosis and active treatment may effectively improve their prognosis. Current treatment options for OSCC include surgery, chemotherapy, biotherapy and radiation therapy (26). However, there are currently no reliable biomarkers to stratify patients and optimize the treatment for OSCC. Therefore, identifying reliable biomarkers and novel molecular targets is critical for stratifying patients to develop precise and personalized treatment regimens. Chronic inflammation is related to the occurrence and development of cancer and may promote cancer progression by activating oncogenic signaling pathways (27).

Wang et al (13) indicated that high expression of TLR4 is conducive to immune escape of tumor cells and promotes the proliferation, invasion and metastasis of tumor cells. MyD88 is an adaptor for the IL-1 and TLR family of downstream inflammatory signaling pathways. In addition, MyD88 signaling has an important role in regulating inflammation during bacterial infection and cancer development (28). Signaling molecules downstream of TLR4/MyD88, for instance NF- $\mathrm{BB}$ and $\mathrm{IKK} / \mathrm{I} \kappa \mathrm{B} / \mathrm{MAPK}$, as well as atypical Akt signaling pathways, are involved in tumor-cell over proliferation (13). According to previous reports that TLR4/MyD88 signaling is dependent on cyclooxygenase, epidermal growth causes excessive proliferation of tumor cells. Activation of TLR4 and MyD88 may increase the expression of prostaglandin E2 and cyclooxygenase-2, enhance EGFR signaling and promote the occurrence and development of inflammation-related cancer (29).

In various studies, different tumor models have been previously used to reach contrasting conclusions. Of note, experimental data associated with TLRS/MyD88 signaling may be contradictory. TLRs have dual functions in tumor development: On the one hand, they may activate the death signal of the tumor; on the other hand, they facilitate proliferation, invasion 
and migration (30). TLR promotes inflammation, primarily through the previously mentioned TLRS/MyD88 signaling pathway. TLR has a tumor-promoting effect when it acts as a proinflammatory factor and has an anti-apoptotic effect. First, TLRs/MyD88 acts as an upstream signaling pathway to regulate the inflammatory pathway. Individuals with chronic inflammation over a long period of time are at a higher risk of developing cancer (31). Furthermore, the TLRs/MyD88 signaling pathway activates $\mathrm{NF}-\kappa \mathrm{B}$, which controls the expression of anti-apoptotic genes and has a strong anti-apoptotic effect (32). High expression of TLR and MyD88 contribute to proliferation, invasion and metastasis (33). Another study suggested that genetically engineered TLR4 overexpression increases the susceptibility of mice to inflammation-induced neoplasia (15).

Furthermore, the TLRs/MyD88 signaling pathway has been reported to have antitumor effects. In clinical trials, patients with high TLR4 expression had a 36.9\% higher decreased risk of cancer-associated death within 5 years (32). Studies have indicated that TLRs and the promotion of downstream mediators may transform the immunomodulatory effects of TLRs/MyD88 into anti-tumor effects $(34,35)$.

The role of TLR as an anti-tumor or pro-tumor agent depends on the type of TLR, different tumor subtypes and the environment of tumor cells. Activation of the TLRs/MyD88 signaling pathway promotes the secretion of IFN and pro-inflammatory cytokines, enhances the antigen presentation ability of dendritic cells (DC) and has anticancer effects (36). Pro-inflammatory cytokines and the enhancement of the antigen presentation ability of DC have anticancer effects, which eventually lead to the maturation of DCs and the enhancement of the antigen presentation ability of DCs (37).

In the present study, a significant increase in the expression of TLR4 and MyD88 in OSCC was observed. This result is consistent with the results of Sharma and Bala (38).The TLR4/MyD88 signaling pathway activates the expression of $N F-\kappa B(39)$ and secondarily promotes the expression of pro-inflammatory genes, thereby triggering the inflammatory response of the host. When activated by TLR4 through stress, injury, death of cells or degradation of extracellular matrix, two signaling pathways are activated, of which the MyD88-dependent pathway is activated in a series of ways, and finally, the NF- $\mathrm{BB}$ signaling pathway is activated. When cells are exposed to the outside stimulation, such as tissue injury or infection (40), the NF- $\mathrm{NB}$ will be transferred from the cytoplasm into the nucleus to relate gene transcription, thus promote inflammation reaction and immune response (41).

To clarify the specific effects of TLR4 and MyD88 in OSCC, the association between biomolecular expression and clinicopathological features was further examined. The expression of MyD88 was greater in highly differentiated and moderately differentiated OSCC than in poorly differentiated OSCC. This suggests that overexpression of MyD88 is involved in tumor differentiation. The results are consistent with the results of Sharma and Bala (38), who also reported that high expression of TLR4 was associated with deep tumor invasion. He et al (42) indicated that activation of the TLR4-MyD88-NF- $\mathrm{B}$ signaling pathway may stimulate epithelial-mesenchymal transition (EMT), which allows cancer cells to detach from the primary site and then invade lymphatic and/or blood vessels, resulting in loss of epithelial adhesion and polarity of cancer cells and promote tumor metastasis. Multiple biomarkers involved in EMT induction may be future therapeutic targets for oral cancer (43). Studies have suggested that blocking MyD88 signaling significantly improves anorexia and fatigue, decreases weight loss, reduces muscle catabolism and atrophy, decreases systemic and central nervous system inflammation, and ultimately improves survival (44-46).

In addition, high expression of MyD88 was observed in patients with short-term relapse in the present study, but the number of patients with short-term relapse was too low (2 cases); therefore, further study is required to confirm this conclusion. To the best of our knowledge, the present study is the first to demonstrate that MyD88 is highly expressed in patients with OSCC, with underlying disease. However, MyD88 expression is not associated with diabetes $(\mathrm{P}=0.286$; Table $\mathrm{SI})$.

It has been established that the immune system has a leading role in the initiation and maintenance of hypertension. However, the process by which the immune response is initiated has remained to be fully elucidated (47). The innate immune system activates the adaptive immune system through direct molecular interactions and the release of immune mediators (48); components of innate immunity may be involved in the initiation of hypertension. Certain TLRs are associated with hypertension (49). These TLRs utilize MyD88 for intracellular signal transduction and activate a pro-inflammatory cascade. It has been indicated that TLR4 is an important receptor for signal transduction in the innate immune system and may influence various cardiovascular diseases, such as heart failure and hypertension (47).

Angiotensin II (Ang II) is the major effector peptide of the renin-angiotensin system. Ang II type 1 receptor promotes blood pressure regulation by interacting with Ang II (50). Singh et al (47) observed that TLR3 and TLR4 differentially induced hypertension and cardiac hypertrophy by Ang II and MyD88 inhibited the pro-inflammatory hypertensive effects of Ang II.

In the TLR4 signaling pathway, the MyD88-dependent signaling pathway is an important activator of $\mathrm{NF}-\kappa \mathrm{B}$ and its subsequent regulation (51). Agarwal et al (52) demonstrated that the increase of various pro-inflammatory cytokines activates the $\mathrm{NF}-\kappa \mathrm{B}$ signaling pathway and leads to increased production of extracellular reactive oxygen species, resulting in intracellular redox state transfer leading to $N F-\kappa B$ activation and enhancement of the $N F-\kappa B$ signaling pathway. The activation of $N F-\kappa B$ was further upregulated. This vicious cycle eventually leads to the development of hypertension.

Hypertension is frequently associated with impaired glucose tolerance, insulin resistance and obesity. Consequently, numerous individuals with hypertension develop diabetes (53). Hypertension is a recognized risk factor for cardiovascular disease, regardless of diabetes (54). Hypertension is more common in patients with type 2 diabetes, and those who have both hypertension and diabetes are at higher risk of developing cardiovascular disease than those who have either disease alone (55).

The TLR pathway is thought to have a key role in the mechanisms leading to diabetes in human and animal 
models (56). Duparc et al (57) reported that MyD88 has a role in developing glucose intolerance and hepatic steatosis. Androulidaki et al (58) identified MyD88 signaling as an important pathogenic factor in autoimmune diabetes. The MyD88 signaling pathway promotes the development of diabetes in non-obese diabetic mice by accelerating the onset of diabetes.

A clear and unambiguous link exists between diabetes and the incidence of coronary artery disease (58). Given the high incidence of coronary artery disease among patients with diabetes, it is not surprising that diabetic patients are at high risk of developing ischemic cardiomyopathy and heart failure. Although the majority of cases of heart failure in patients with diabetes are caused by progression from coronary artery disease to ischemic cardiomyopathy (59), the long-term metabolic disorders of diabetes may also be toxic to the heart muscle (60). Diabetes mellitus is widely recognized as an important risk factor for the development of heart failure and is an independent risk factor for increased mortality in patients with heart failure (61). Dong et al (62) reported that the TLR4 signaling pathway may lead to myocardial injury in patients with diabetes. Type 2 diabetes and congestive heart failure occur simultaneously due to common risk factors, such as coronary artery disease, and the direct cardiotoxic effects of type 2 diabetes (62). Follow-up data from the Framingham study collected over 20 years indicated that diabetes is an independent risk factor for the development of heart failure (62).

Myocardial inflammation caused by coronary microembolism (CME) is the major cause of cardiac injury (39). Su et al (39) indicated that TLR4/MyD88/NF- $\mathrm{B}$ signaling is involved in myocardial inflammation after CME, activating the NOD-like receptor protein 3 inflammasome, promoting the inflammatory cascade and aggravating myocardial injury. Numerous studies have indicated that the TLR4/MyD88/NF- $\kappa \mathrm{B}$ signaling pathway controls the production of pro-inflammatory factors and induces the inflammatory response of myocardial tissue, which is the major cause of myocardial tissue injury (39). The role of MyD88 (39) and TLR4 (63) has been demonstrated in myocardial infarction and aortic band-induced cardiac hypertrophy.

To sum up, hypertension, heart disease and diabetes affect each other and the TLR4/MyD88 pathway has an important role in the occurrence and development of hypertension, heart disease and diabetes, which may explain the high expression of MyD88 in basic diseases.

Furthermore, the present study also suggested that TLR4 expression levels were significantly associated with tumor growth time; a longer tumor growth time was linked to a higher TLR4 expression level. These results prove that TLR4 is closely related to the occurrence and development of OSCC. TLR4 may be involved in and promote the development of cancer, and its high expression may indicate a poor prognosis for OSCC.These results further confirm the previous assumption that TLR4 and MyD88 are related to tumor progression.

A study by Park et al (64) on inflammation suggested that lipopolysaccharide was able to regulate the oligomerization of TLR4. It binds to five TIR domains, including MyD88 (65). Among them, the combination of TLR4 and MyD88 is able to initiate $\mathrm{NF}-\kappa \mathrm{B}$ and thus upregulate pro-inflammatory genes to activate the inflammatory response to infection (66). In the present study, the expression levels of TLR4 and MyD88 were determined to be closely correlated in OSCC. Combined with those of previous studies, the present results indicated that TLR4 also regulates MyD88 in oral cancer.

Existing studies reported on numerous inhibitors targeting TLR4 and MyD88. Eritoran (E5564) (67) is an investigational drug for treating severe sepsis and acts as an inhibitor of TLR4. Curcumin, a polyphenol, suppresses the TLR4/MAPK/NF- $\kappa$ B pathway to inhibit excessive inflammatory mediators (68). Reversed retinoic acid targets $\mathrm{TNF}-\alpha$ and hydrogen oxide synthase 2 inhibitors through TLR4/NF- $\kappa \mathrm{B}$ signaling pathways. This may resemble a novel treatment strategy for oral cancer (69).

In the present study, TLR4 and MyD88 were only assessed at the tissue and molecular levels and the analysis of TLR4 and MyD88 remains insufficient. Both of them involve complex networks and various pathways, which should be improved by experiments at the cellular and animal levels, which is a goal of future research by our group. The results of the present study are based on 2D cross-sections, while 3D organotypic models are receiving increasing attention due to their ability to reconstruct precise tissue. Human physiology is profoundly different from the mouse model system. Common drugs such as ibuprofen (70) and warfarin (71), which are metabolized differently in the livers of humans and rodents, where ibuprofen is used to treat pain, fever and inflammation and warfarin is used as an anticoagulant, are both toxic to mice. It is not surprising that there are large metabolic differences between human and laboratory models. Most drug candidates do not enter the market due to unrepresentative two-dimensional preclinical models and poor correlation between preclinical and clinical trial results. Even when drugs are approved for clinical use, certain products may be recalled due to serious adverse reactions. 3D tissue cultures may produce samples suitable for low-cost, high-throughput drug screening and predict human drug responses in vitro in a timely manner.

Organ on a chip is a promising tool for the preclinical testing of drugs. Tumors have a complex microenvironment, including a dense extracellular matrix and a wide variety of stromal cells and immune cells (72). Irregular blood vessels and limited perfusion of nutrients may significantly affect the efficacy of administration therapy. By combining 3D organic culture systems with microfluidics to form an 'organ on a chip', in vitro controlled microfluidics are able to stimulate blood flow within the organ and reproduce the dynamic distribution of nutrients (73). By connecting different organs on a chip, microfluidic systems may simulate complex multi-organ metabolism and pharmacokinetics of drugs. Cellular behavior, particularly drug responses, may be easily monitored. The organ-on-a-chip approach has been used to model various healthy and diseased organs, such as kidney (74) and lung (75).

The quasi-human organ is a three-dimensional culture system derived from stem cells that have the potential to reconstruct the structure and physiology of human organs in remarkable detail. Human organoids are already being used to study infectious diseases, genetic diseases and cancer through genetic engineering of human stem cells, and may also be generated directly from a patient's biopsy sample (76). 
3D culture systems are highly similar to actual human organs and in certain cases, they are histologically indistinguishable. A feature common to all organoids is that they are composed of pluripotent stem cells or adult stem cells (also known as tissue stem cells), mimicking human development or organ regeneration in vitro. Thus, analysis of the formation of organ-like systems may provide valuable information on the mechanisms of human development and organ regeneration, highlighting its value in basic biological research and its potential applications in drug testing and molecular medicine.

Establishing an animal model for a particular disease requires prior knowledge of the pathogenic conditions or genes involved. Organ models may be generated directly from affected patients without prior knowledge of the specific genes involved. This is particularly relevant to polygenetic diseases (such as inflammatory bowel disease, if the pathology is caused by the affected epithelial cells) and cancer, where cancer-like organs may be isolated directly from the patient (77). One drawback of organ-like systems is the lack of inter-organ communication. There is the possibility of generating a cavity device that combines organic research with organ-on-a-chip, allowing different organ-like types to be cultivated separately, thereby preventing organ-like uncontrolled fusion while allowing organ-like communication $(78,79)$. Human organ-like systems will offer unprecedented opportunities to improve human health.

In conclusion, the present study confirmed differences in TLR4 and MyD88 expression between OSCC and paracancerous tissues and analyzed the relationship between TLR4 and MyD88, as well as clinical pathology parameters and patient prognosis. It was demonstrated for the first time that MyD88 is overexpressed in patients with underlying conditions, such as hypertension and diabetes, and may have an important role in long-term prognosis. Furthermore, TLR4 expression levels were significantly correlated with the tumor growth time. These gene expression experiments verified that MyD88 and TLR4 may be used as biomarkers, that may provide guidance for decision making regarding the treatment of OSCC.

\section{Acknowledgements}

The authors would like to thank Ms. Danping Li (Pathology of the School of Stomatology, Guangxi Medical University, Guangxi, China) for helping prepare the tissue sections. The authors would also like to thank Mr. Qincao Tang (Department of Oral and Maxillofacial Surgery, Guangxi Medical University, Guangxi, China) for technical support with RT-qPCR.

\section{Funding}

The present study was supported by the National Natural Science Foundation of China (grant no. 81560187).

\section{Availability of data and materials}

The datasets used and/or analyzed during the current study are available from the corresponding author upon reasonable request.

\section{Authors' contributions}

LL was involved in designing the study and participated in the collection of specimens, performing the experiments, data analysis, interpretation of the data and drafting and revising the manuscript. ZZ and KM assisted in interpreting experimental results and participated in manuscript writing. PL assisted in performing the experiments and the completion of interpreting the research results and assisted in revising the manuscript. ZW and $\mathrm{YW}$ assisted in the collection of specimens and revision of the manuscript. $\mathrm{YC}$ and $\mathrm{XM}$ provided assistance in performing the experiments. TZ assisted with the data analysis and revision of the manuscript. DW conceived and directed the study and assisted with interpreting the study results and the revision of the manuscript. LL and ZZ authenticated all the raw data. All authors have read and approved the final manuscript.

\section{Ethics approval and consent to participate}

The present study was approved by the Ethics Committee of the College of Stomatology, Guangxi Medical University (Nanning, China).

\section{Patient consent for publication}

Not applicable.

\section{Competing interests}

The authors declare that they have no competing interests.

\section{References}

1. de Groen RAL, Schrader AMR, Kersten MJ, Pals ST and Vermaat JSP: MYD88 in the driver's seat of B-cell lymphomagenesis: From molecular mechanisms to clinical implications. Haematologica 104: 2337, 2019.

2. Ali J, Sabiha B, Jan HU, Haider SA, Khan AA and Ali SS: Genetic etiology of oral cancer. Oral Oncol 70: 23-28, 2017.

3. Oh HN, Seo JH, Lee MH, Kim C, Kim E, Yoon G, Cho SS, Cho YS, Choi HW, Shim JH and Chae JI: Licochalcone C induced apoptosis in human oral squamous cell carcinoma cells by regulation of the JAK2/STAT3 signaling pathway. J Cell Biochem 119: 10118-10130, 2018.

4. Rivera C: Essentials of oral cancer. Int J Clin Exp Pathol 8: 11884-11894, 2015.

5. Edwards BK, Brown ML, Wingo PA, Howe HL, Ward E, Ries LA, Schrag D, Jamison PM, Jemal A, Wu XC, et al: Annual report to the nation on the status of cancer, 1975-2002, featuring population-based trends in cancer treatment. J Natl Cancer Inst 97: 1407-1427, 2005.

6. Lindemann A, Takahashi H, Patel AA, Osman AA and Myers JN: Targeting the DNA damage response in OSCC with TP 53 mutations. J Dent Res 97: 635-644, 2018.

7. Roi A, Roi CI, Negruțiu ML, Riviș M, Sinescu C and Rusu LC: The challenges of OSCC diagnosis: Salivary cytokines as potential biomarkers. J Clin Med 9: 2866, 2020.

8. Chen R, Alvero AB, Silasi DA and Mor G: Inflammation, cancer and chemoresistance: Taking advantage of the toll-like receptor signaling pathway. Am J Reprod Immunol 57: 93-107, 2007.

9. Mehmeti M, Allaoui R, Bergenfelz C, Saal LH, Ethier SP, Johansson ME, Jirström K and Leandersson K: Expression of functional toll like receptor 4 in estrogen receptor/progesterone receptor-negative breast cancer. Breast Cancer Res 17: 130, 2015.

10. Semlali A, Reddy Parine N, Arafah M, Mansour L, Azzi A, Al Shahrani O, Al Amri A, Shaik JP, Aljebreen AM, Alharbi $\mathrm{O}$, et al: Expression and polymorphism of toll-like receptor 4 and effect on NF-kB mediated inflammation in colon cancer patients. PLoS One 11: e0146333, 2016. 
11. Takazawa Y, Kiniwa Y, Ogawa E, Uchiyama A, Ashida A, Uhara $\mathrm{H}$, Goto $\mathrm{Y}$ and Okuyama R: Toll-like receptor 4 signaling promotes the migration of human melanoma cells. Tohoku J Exp Med 234: 57-65, 2014

12. Luo XZ, He QZ and Wang K: Expression of toll-like receptor 4 in ovarian serous adenocarcinoma and correlation with clinical stage and pathological grade. Int J Clin Exp Med 8: 14323-14327, 2015.

13. Wang JQ, Jeelall YS, Ferguson LL and Horikawa K: Toll-like receptors and cancer: MYD88 mutation and inflammation. Front Immunol 5: 367, 2014.

14. Deguine J and Barton GM: MyD88: A central player in innate immune signaling. F1000Prime Rep 6: 97, 2014

15. Wang EL, Qian ZR, Nakasono M, Tanahashi T, Yoshimoto K, Bando Y, Kudo E, Shimada M and Sano T: High expression of toll-like receptor $4 /$ myeloid differentiation factor 88 signals correlates with poor prognosis in colorectal cancer. Br J Cancer 102: 908-915, 2010.

16. Huang HY, Zhang ZJ, Cao CB, Wang N, Liu FF, Peng JQ, Ren XJ and Qian J: The TLR4/NF- $\kappa \mathrm{B}$ signaling pathway mediates the growth of colon cancer. Eur Rev Med Pharmacol Sci 18 3834-3843, 2014

17. Chen X, Zhao F, Zhang H, Zhu Y, Wu K and Tan G: Significance of TLR4/MyD88 expression in breast cancer. Int J Clin Exp Pathol 8: 7034-7039, 2015.

18. Mills SE, Carter D, Greenson JK, Reuter VE and Stoler MH: Sternberg's diagnostic surgical pathology, 5th edition. Vol. 2-2. Lippincott Williams \& Wilkins, Philidelphia, PA, USA, pp 2348 2348,2012

19. The Union for International Cancer Control (UICC): TNM History, Evolution and Milestones. https://www.uicc.org/ sites/main/files/atoms/files/TNM-History-2021.pdf. Accessed September 11, 2021.

20. Lin SC, Lo YC and Wu H: Helical assembly in the MyD88-IRAK4-IRAK2 complex in TLR/IL-1R signalling. Nature 465: 885-890, 2010.

21. Song Y, Zhou M, Cao Y, Qi J, Geng J and Liu X: Expression of GLP-1 receptor and CD26 in human thyroid C-cells: The association of thyroid C-cell tumorigenesis with incretin-based medicine. Oncol Lett 13: 2684-2690, 2017.

22. Torre LA, Bray F, Siegel RL, Ferlay J, Lortet-Tieulent J and Jemal A: Global cancer statistics, 2012. CA Cancer J Clin 65: 87-108, 2015.

23. González-Moles MÁ, Warnakulasuriya S, González-Ruiz I, González-Ruiz L, Ayén Á, Lenouvel D, Ruiz-Ávila I and Ramos-García P: Clinicopathological and prognostic characteristics of oral squamous cell carcinomas arising in patients with oral lichen planus: A systematic review and a comprehensive meta-analysis. Oral Oncol 106: 104688, 2020.

24. Warnakulasuriya S, Johnson NW and Van der Waal I: Nomenclature and classification of potentially malignant disorders of the oral mucosa. J Oral Pathol Med 36: 575-580, 2007.

25. Güneri $P$ and Epstein JB: Late stage diagnosis of oral cancer: Components and possible solutions. Oral Oncol 50: 1131-1136, 2014.

26. Cheng YS, Rees T and Wright J: A review of research on salivary biomarkers for oral cancer detection. Clin Transl Med 3: 3, 2014

27. Rivera C: The challenge of the state of susceptibility to oral cancer. J Oral Res 4: 8-9, 2015

28. Sakurai T, Kashida H, Watanabe T, Hagiwara S, Mizushima T, Iijima H, Nishida N, Higashitsuji H, Fujita J and Kudo M: Stress response protein cirp links inflammation and tumorigenesis in colitis-associated cancer. Cancer Res 74: 6119-6128, 2014.

29. Oshima $\mathrm{H}$ and Oshima $\mathrm{M}$ : The inflammatory network in the gastrointestinal tumor microenvironment: Lessons from mouse models. J Gastroenterol 47: 97-106, 2012.

30. Wang L, Zhu R, Huang Z, Li $\mathrm{H}$ and $\mathrm{Zhu} \mathrm{H}$ : Lipopolysaccharide-induced toll-like receptor 4 signaling in cancer cells promotes cell survival and proliferation in hepatocellular carcinoma. Dig Dis Sci 58: 2223-2236, 2013.

31. Coussens LM and Werb Z: Inflammation and cancer. Nature 420 : 860-867, 2002

32. Fukata M, Chen A, Klepper A, Krishnareddy S, Vamadevan AS, Thomas LS, Xu R, Inoue H, Arditi M, Dannenberg AJ and Abreu MT: Cox-2 is regulated by toll-like receptor-4 (TLR4) signaling: Role in proliferation and apoptosis in the intestine. Gastroenterology 131: 862-877, 2006.

33. Sahu U, Choudhury A, Parvez S, Biswas S and Kar S: Induction of intestinal stemness and tumorigenicity by aberrant internalization of commensal non-pathogenic E. coli. Cell Death Dis 8: e2667, 2017.
34. Paarnio K, Väyrynen S, Klintrup K, Ohtonen P, Mäkinen MJ, Mäkelä J and Karttunen TJ: Divergent expression of bacterial wall sensing toll-like receptors 2 and 4 in colorectal cancer. World J Gastroenterol 23: 4831-4838, 2017.

35. Yu L, Wang L and Chen S: Dual character of toll-like receptor signaling: Pro-tumorigenic effects and anti-tumor functions. Biochim Biophys Acta 1835: 144-154, 2013

36. Mikulandra M, Pavelic J and Glavan TM: Recent findings on the application of toll-like receptors agonists in cancer therapy. Curr Med Chem 24: 2011-2032, 2017.

37. Kubo T, Hatton RD, Oliver J, Liu X, Elson CO and Weaver CT: Regulatory $\mathrm{T}$ cell suppression and anergy are differentially regulated by proinflammatory cytokines produced by TLR-activated dendritic cells. J Immunol 173: 7249-7258, 2004.

38. Sharma Y and Bala K: Role of toll like receptor in progression and suppression of oral squamous cell carcinoma. Oncol Rev 14: 456,2020

39. Su Q, Li L, Sun Y, Yang H, Ye Z and Zhao J: Effects of the TLR4/Myd88/NF- $\mathrm{BB}$ signaling pathway on NLRP3 inflammasome in coronary microembolization-induced myocardial injury. Cell Physiol Biochem 47: 1497-1508, 2018.

40. Lawrence T: The nuclear factor NF-kappaB pathway in inflammation. Cold Spring Harb Perspect Biol 1: a001651, 2009.

41. Qin DP, Sun PN, Zhou YJ, Chen FM, Zhang CL, Han JX and Yang XJ: Effect of tripterygium wilfordii polycoride upon inflammation and TLR4/MyD88 signaling pathway in ulcerative colitis rats model. Zhonghua Yi Xue Za Zhi 96: 1444-1449, 2016 (In Chinese)

42. He Z, Deng R, Huang X, Ni Y, Yang X, Wang Z and Hu Q: Lipopolysaccharide enhances OSCC migration by promoting epithelial-mesenchymal transition. J Oral Pathol Med 44: 685-692, 2015.

43. Todosi AM, Gavrilescu MM, Aniţei GM, Filip B and Scripcariu V: Colon cancer at the molecular level-usefulness of epithelial-mesenchymal transition analysis. Rev Med Chir Soc Med Nat Iasi 116: 1106-1111, 2012

44. Zhu X, Burfeind KG, Michaelis KA, Braun TP, Olson B, Pelz KR, Morgan TK and Marks DL: MyD88 signalling is critical in the development of pancreatic cancer cachexia. J Cachexia Sarcopenia Muscle 10: 378-390, 2019.

45. Zhang Y and Zeng Y: Curcumin reduces inflammation in knee osteoarthritis rats through blocking TLR4/MyD88/NF- $\mathrm{KB}$ signal pathway. Drug Dev Res 80: 353-359, 2019.

46. Ju M, Liu B, He H, Gu Z, Liu Y, Su Y, Zhu D, Cang J and Luo Z: MicroRNA-27a alleviates LPS-induced acute lung injury in mice via inhibiting inflammation and apoptosis through modulating TLR4/MyD88/NF- $\mathrm{B}$ pathway. Cell Cycle 17: 2001-2018, 2018

47. Singh MV, Cicha MZ, Nunez S, Meyerholz DK, Chapleau MW and Abboud FM: Angiotensin II-induced hypertension and cardiac hypertrophy are differentially mediated by TLR3- and TLR4-dependent pathways. Am J Physiol Heart Circ Physiol 316: H1027-H1038, 2019.

48. Jain A and Pasare C: Innate control of adaptive immunity: Beyond the three-signal paradigm. J Immunol 198: 3791-3800, 2017.

49. Dange RB, Agarwal D, Teruyama R and Francis J: Toll-like receptor 4 inhibition within the paraventricular nucleus attenuates blood pressure and inflammatory response in a genetic model of hypertension. J Neuroinflammation 12: 31, 2015.

50. Kang YM, Zhang DM, Yu XJ, Yang Q, Qi J, Su Q, Suo YP, Yue LY, Zhu GQ and Qin DN: Chronic infusion of enalaprilat into hypothalamic paraventricular nucleus attenuates angiotensin II-induced hypertension and cardiac hypertrophy by restoring neurotransmitters and cytokines. Toxicol Appl Pharmacol 274: 436-444, 2014.

51. Zhu HT, Bian C, Yuan JC, Chu WH, Xiang X, Chen F, Wang CS, Feng $\mathrm{H}$ and Lin JK: Curcumin attenuates acute inflammatory injury by inhibiting the TLR4/MyD88/NF- $\kappa$ B signaling pathway in experimental traumatic brain injury. J Neuroinflammation 11 : 59, 2014.

52. Agarwal D, Welsch MA, Keller JN and Francis J: Chronic exercise modulates RAS components and improves balance between pro- and anti-inflammatory cytokines in the brain of SHR. Basic Res Cardiol 106: 1069-1085, 2011.

53. Gress TW, Nieto FJ, Shahar E, Wofford MR and Brancati FL: Hypertension and antihypertensive therapy as risk factors for type 2 diabetes mellitus. Atherosclerosis risk in communities study. N Engl J Med 342: 905-912, 2000. 
54. Bensimhon HF and Cavender MA: Hypertension treatment in diabetes: Focus on heart failure prevention. Heart Fail Clin 15: 551-563, 2019.

55. Stamler J, Vaccaro O, Neaton JD and Wentworth D: Diabetes, other risk factors, and 12-yr cardiovascular mortality for men screened in the multiple risk factor intervention trial. Diabetes Care 16: 434-444, 1993.

56. Chervonsky A: Innate receptors and microbes in induction of autoimmunity. Curr Opin Immunol 21: 641-647, 2009.

57. Duparc T, Plovier H, Marrachelli VG, Van Hul M, Essaghir A, Ståhlman M, Matamoros S, Geurts L, Pardo-Tendero MM, Druart C, et al: Hepatocyte MyD88 affects bile acids, gut microbiota and metabolome contributing to regulate glucose and lipid metabolism. Gut 66: 620-632, 2017.

58. Androulidaki A, Wachsmuth L, Polykratis A and Pasparakis M: Differential role of MyD88 and TRIF signaling in myeloid cells in the pathogenesis of autoimmune diabetes. PLoS One 13 e0194048, 2018.

59. Marwick TH: Diabetic heart disease. Postgrad Med J 84 188-192, 2008.

60. Marwick TH, Ritchie R, Shaw JE and Kaye D: Implications of underlying mechanisms for the recognition and management of diabetic cardiomyopathy. J Am Coll Cardiol 71: 339-351, 2018.

61. Mitchell JB, Bubolz T, Paul JE, Pashos CL, Escarce JJ, Muhlbaier LH, Wiesman JM, Young WW, Epstein RS and Javitt JC: Using medicare claims for outcomes research. Med Care 32 (Suppl 7): JS38-JS51, 1994.

62. Dong B, Qi D, Yang L, Huang Y, Xiao X, Tai N, Wen L and Wong FS: TLR4 regulates cardiac lipid accumulation and diabetic heart disease in the nonobese diabetic mouse model of type 1 diabetes. Am J Physiol Heart Circ Physiol 303: H732-H742, 2012.

63. Jiang DS, Zhang XF, Gao L, Zong J, Zhou H, Liu Y, Zhang Y, Bian ZY, Zhu LH, Fan GC, et al: Signal regulatory protein- 0 protects against cardiac hypertrophy via the disruption of toll-like receptor 4 signaling. Hypertension 63: 96-104, 2014

64. Park BS, Song DH, Kim HM, Choi BS, Lee H and Lee JO The structural basis of lipopolysaccharide recognition by the TLR4-MD-2 complex. Nature 458: 1191-1195, 2009.

65. O'Neill LA and Bowie AG: The family of five: TIR-domain-containing adaptors in toll-like receptor signalling. Nat Rev Immunol 7: 353-364, 2007.

66. Blauvelt A, Lebwohl MG and Bissonnette R: IL-23/IL-17A dysfunction phenotypes inform possible clinical effects from anti-IL-17A therapies. J Invest Dermatol 135: 1946-1953, 2015.

67. Shirey KA, Lai W, Scott AJ, Lipsky M, Mistry P, Pletneva LM, Karp CL, McAlees J, Gioannini TL, Weiss J, et al: The TLR4 antagonist Eritoran protects mice from lethal influenza infection. Nature 497: 498-502, 2013.

68. Xu Z, Ren T, Xiao C, Li H and Wu T: Nickel promotes the invasive potential of human lung cancer cells via TLR4/MyD88 signaling. Toxicology 285: 25-30, 2011.
69. Feng X, Tan W, Cheng S, Wang H, Ye S, Yu C, He Y, Zeng J, Cen J, Hu J, et al: Upregulation of microRNA-126 in hepatic stellate cells may affect pathogenesis of liver fibrosis through the NF- $\kappa$ B pathway. DNA Cell Biol 34: 470-480, 2015.

70. Sanoh S, Horiguchi A, Sugihara K, Kotake Y, Tayama Y, Uramaru N, Ohshita H, Tateno C, Horie T, Kitamura S and Ohta S: Predictability of metabolism of ibuprofen and naproxen using chimeric mice with human hepatocytes. Drug Metab Dispos 40: 2267-2272, 2012.

71. Inoue T, Nitta K, Sugihara K, Horie T, Kitamura S and Ohta S: CYP2C9-catalyzed metabolism of S-warfarin to 7-hydroxywarfarin in vivo and in vitro in chimeric mice with humanized liver. Drug Metab Dispos 36: 2429-2433, 2008.

72. Sherman MH, Yu RT, Tseng TW, Sousa CM, Liu S, Truitt ML, He N, Ding N, Liddle C, Atkins AR, et al: Stromal cues regulate the pancreatic cancer epigenome and metabolome. Proc Natl Acad Sci USA 114: 1129-1134, 2017.

73. Sun W, Luo Z, Lee J, Kim HJ, Lee K, Tebon P, Feng Y, Dokmeci MR, Sengupta S and Khademhosseini A: Organ-ona-chip for cancer and immune organs modeling. Adv Healthc Mater 8: 1801363, 2019.

74. Musah S, Mammoto A, Ferrante TC, Jeanty SSF, Hirano-Kobayashi M, Mammoto T, Roberts K, Chung S, Novak R, Ingram M, et al: Mature induced-pluripotentstem-cell-derived human podocytes reconstitute kidney glomerular-capillary-wall function on a chip. Nat Biomed Eng 1: 0069, 2017.

75. Huh D, Matthews BD, Mammoto A, Montoya-Zavala M, Hsin HY and Ingber DE: Reconstituting organ-level lung functions on a chip. Science 328: 1662-1668, 2010.

76. Kim J, Koo BK and Knoblich JA: Human organoids: Model systems for human biology and medicine. Nat Rev Mol Cell Biol 21: 571-584, 2020.

77. Dotti I, Mora-Buch R, Ferrer-Picón E, Planell N, Jung P, Masamunt MC, Leal RF, Martín de Carpi J, Llach J, Ordás I, et al: Alterations in the epithelial stem cell compartment could contribute to permanent changes in the mucosa of patients with ulcerative colitis. Gut 66: 2069-2079, 2017.

78. Gonneaud A, Asselin C, Boudreau F and Boisvert FM: Phenotypic analysis of organoids by proteomics. Proteomics 17, 2017.

79. Akhtar AA, Sances S, Barrett R and Breunig JJ: Organoid and organ-on-a-chip systems: New paradigms for modeling neurological and gastrointestinal disease. Curr Stem Cell Rep 3: 98-111, 2017

This work is licensed under a Creative Commons Attribution-NonCommercial-NoDerivatives 4.0 International (CC BY-NC-ND 4.0) License. 